\title{
La artrodesis de las cuatro esquinas con placa circular
}

\author{
A. García López ${ }^{(1)}$, L. Clavel Rojo ${ }^{(2)}$, D. Perea Tortosa ${ }^{(2)}$, \\ J. Hernández López ${ }^{(2)}$, A. Aguirre Pastor ${ }^{(2)}$ \\ (1) FEA de COyt. Servicio de Traumatología y Cirugía Ortopédica. Hospital General \\ UNIVERSITARIO DE ALICANTE \\ (2) MiR. Servicio de Traumatología y Cirugía Ortopédica. Hospital General Universitario de ElChe
}

\author{
Correspondencia: \\ Dr. Antonio García López \\ C/ Madre Teresa de Calcuta, 4, bloque 4, esc. 2, 4으 I \\ 03016 Alicante \\ e-mail: garcialopez1@hotmail.com
}

Objetivo: La artrodesis de las cuatro esquinas del carpo fue descrita inicialmente para el tratamiento del colapso avanzado del carpo e incluye la extirpación del escafoides y la fusión de los huesos semilunar, piramidal, hueso grande y ganchoso. Se ha utilizado la fijación con placas circulares con el objetivo de conseguir una fijación estable inmediata. El propósito de este estudio es evaluar la efectividad de estas placas en esta artrodesis mediocarpiana y valorar sus indicaciones así como ciertas consideraciones técnicas.

Material y métodos: En este estudio se ha incluido una serie de 20 pacientes intervenidos para practicar una artrodesis de las cuatro esquinas con placa SpiderÒ que presentaban un estadio II ó III de proceso degenerativo avanzado del carpo. También se ha utilizado esta técnica para otras indicaciones como las lesiones osteocondrales de la cabeza del hueso grande, necrosis avascular de la cabeza del hueso grande, artrosis mediocarpiana o inestabilidad mediocarpiana. El seguimiento medio ha sido de 18 meses $(\mathrm{R}=8-25)$. Resultados y conclusiones: Se ha conseguido el $100 \%$ de consolidaciones, y una importante disminución del dolor en todos los casos, manteniéndose una flexión palmar postquirúrgica de $34^{\circ}\left(\mathrm{R}=12-45^{\circ}\right)$; una flexión dorsal de $29^{\circ}\left(\mathrm{R}=12-45^{\circ}\right)$; una desviación radial postoperatoria de $12^{\circ}\left(\mathrm{R}=0-16^{\circ}\right)$ y una desviación cubital de $17^{\circ}\left(\mathrm{R}=0-20^{\circ}\right)$. Se apreció un aumento de la fuerza de agarre tras la cirugía y todos los pacientes volvieron a sus actividades previas.

Esta técnica quirúrgica ofrece una fijación estable inmediata que permite una movilización precoz tras la cirugía. Es importante el relleno de injerto de escafoides o cresta iliaca en los espacios entre los huesos a artrodesar.

Palabras clave: Artrodesis, artrosis carpiana, тиñeca.
Purpose: The four-bone fusion, which combines scaphoid resection with arthrodesis of capitate, hamate, lunate and triquetrum, was first described for using in carpi advanced collapse. With the aim of achieving immediate stable fixation, we have fix the four bone fusion mass with a circular plate. The aim of this study was to assess the effectiveness of circular plates in midcarpal arthrodesis and provide some considerations about the surgical technique and indications.

Material and Methods: We studied 20 patients with advanced stage II and III advanced collapse of carpus treated by midcarpal arthrodesis with a SpiderÒ circular plates. Also we have used this technique in head of capitate osteocondral lesion, capitate avascular necrosis, midcarpal osteoarthritis and midcarpal instability. Mean follow-up was 18 month $(\mathrm{R}=$ 8-25 month).

Results and conclusions: Bone fusion and a significant decrease in pain was seen in all cases. Postoperative flexion was $34^{\circ}\left(\mathrm{R}=12-45^{\circ}\right)$; extension $29^{\circ}\left(\mathrm{R}=12-45^{\circ}\right)$; there was postoperative radial desviation of $12^{\mathrm{o}}\left(\mathrm{R}=0-16^{\circ}\right)$ and ulnar desviation of $17^{\circ}\left(\mathrm{R}=0-20^{\circ}\right)$. After surgery an increase in grip strength was seen and all the patients were able to return to their previous activities and jobs.

In conclusion, this technique offers inmediate stable fixation which allows early mobilization and a high union rate. But it is also very important to fill the spaces between the carpal bones with bone chips from the iliac crest.

Key words: arthodesis, carpal osteoarthritis, wrist. 


\section{INTRODUCCIÓN}

La artrodesis de las cuatro esquinas fue inicialmente descrita por Watson et al. para el tratamiento del colapso avanzado del carpo en sus dos patrones fundamentales: disociativos o scapho-lunate advanced collapse (SLAC) y secundarios a seudoartrosis de escafoides o scaphoid non-union advanced colapse (SNAC) en las fases II y III ${ }^{1}$. Combina la extirpación del escafoides carpiano con la artrodesis de los huesos grande, ganchoso, semilunar y piramidal. Para la fijación de los huesos a fusionar se han utilizado tornillos de Herbert y grapas realizando una configuración en $\mathrm{U}$, pero lo más extendido es el uso de agujas de Kirchsner. Diseñamos una modificación a esta técnica con el objetivo de conseguir un alto índice de consolidaciones y realizar una fijación estable que permitiese una rehabilitación precoz, y por tanto mantener el mayor arco de movilidad posible. Esta modificación se caracteriza por el uso de un injerto atornillado corticoesponjoso procedente de la cresta ilíaca y el relleno del defecto creado en el carpo con injerto esponjoso. ${ }^{2}$ Posteriormente se introdujeron en el mercado placas circulares para la estabilización de esta artrodesis y comenzamos a utilizarlas. El presente estudio pretende evaluar nuestros resultados obtenidos con el uso de la placa circular de fusión limitada carpiana SpiderÒ (Kinetikos Medical Inc., San Diego, CA).

\section{MATERIAL Y MÉTODO}

El estudio incluye 20 pacientes intervenidos para practicar una artrodesis de las 4 esquinas con placa circular. La serie consiste en 16 varones y 4 mujeres con una edad media en el momento de la cirugía de 54 años $(\mathrm{R}=26-81)$. En 15 pacientes estaba afectada la mano dominante. Eran trabajadores manuales 18 de los 20 pacientes

El diagnóstico original fue 9 muñecas SNAC, 5 muñecas SLAC, 2 necrosis del hueso grande, 2 inestabilidades mediocarpianas y 2 secuelas postraumáticas (luxaciones perilunares). En estas últimas la artrodesis se realizó en un caso de luxación transescafoperilunar, en el

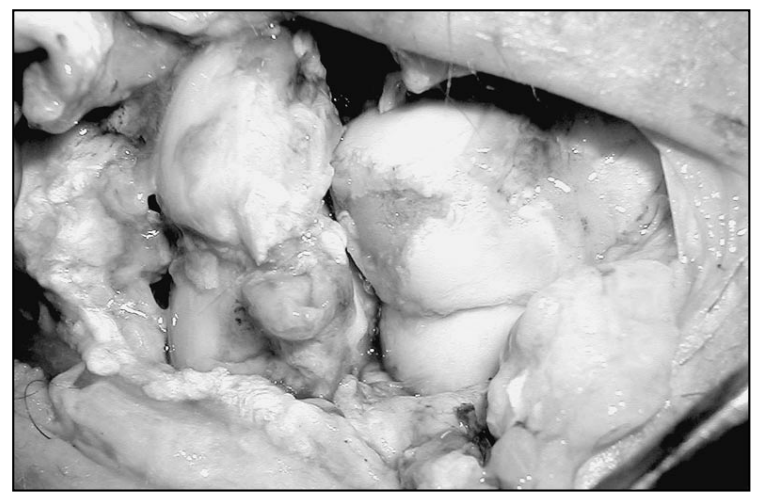

Figura 1. Campo quirúrgico de un abordaje dorsal de una luxación transescafoperilunar donde se observa una gran lesión osteocondral que precisó una artrodesis de las 4 esquinas.

momento agudo, cuando se evidenció durante la cirugía una gran lesión osteocondral en la cabeza del hueso grande (Figura 1), el otro caso tras la evolución de una luxación del semilunar reducida y sintetizada que desarrolló artrosis mediocarpiana. Uno de los casos de seudoartrosis de escafoides carpiano había sido tratado con una prótesis de escafoides de silicona que había llevado durante 20 años hasta desarrollar un patrón destructivo en el carpo, que respetaba la articulación radio-semilunar. Todos los pacientes tratados en fase crónica presentaban sintomatología dolorosa. De los 14 casos con colapso avanzado del carpo solo 8 recordaban un antecedente traumático claro; el tiempo medio desde el traumatismo hasta la cirugía fue de 18,8 años $(\mathrm{R}=12-30)$.

Todos los pacientes referían intenso dolor que les incapacitaba para realizar su actividad habitual, fueron evaluados mediante la escala visual analógica del dolor (VAS), puntuando 0 como ausencia de dolor y 10 como el máximo dolor soportable.

Se midió la movilidad con un goniómetro, la flexión palmar; flexión dorsal; desviación radial y cubital, pre y postoperatoria, y la fuerza con un dinamómetro Baseline ${ }^{\circledR}$, empleando la media de tres determinaciones.

Las pruebas de imagen incluyeron radiografías anteroposteriores y laterales de ambas muñecas, valorándose el grado de consolidación de la artrodesis, la posición de la placa y los tornillos y el índice de altura del carpo, definido como tal el cociente obtenido entre la distancia comprendida entre la superficie articular 


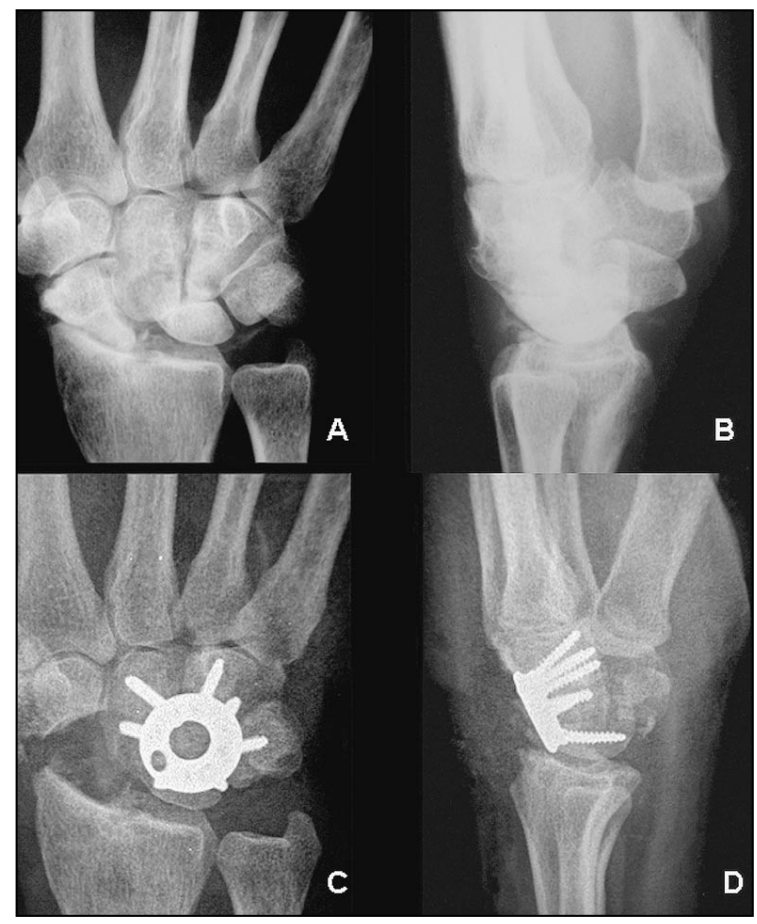

Figura 2. A y B: Muñeca SLAC, radiología preoperatorio con intensa artrosis periescafoidea y escafoides muy escleroso, que no permite una fuente adecuada de injerto. C y D: Radiología postoperatoria donde se observa que se ha realizado una extirpación completa del escafoides carpiano y se ha obtenido la consolidación.

del radio al tercer metacarpiano y la longitud del tercer metacarpiano ${ }^{3}$. Se considera que la artrodesis está consolidada radiológicamente cuando existe paso de trabéculas, cuando no hay rotura del material de osteosíntesis y existe buena integración del material de osteosíntesis sin osteolisis alrededor de los tornillos. No se hizo TAC de control sistemático salvo en situaciones dudosas para evidenciar la consolidación.

\section{TÉCNICA QUIRÚRGICA}

Se realiza una incisión longitudinal dorsal de unos $7 \mathrm{~cm}$. entre el tercer y cuarto compartimentos extensores, transponiendo radialmente el tendón del extensor largo del pulgar para exponer la capsula articular. Se identifica el nervio interóseo posterior que se secciona proximalmente. Se abre la cápsula articular longitudinalmente y si es necesario se realiza la extirpación del escafoides con una gubia, teniendo cuidado de no dañar los ligamentos extrínsecos radiocarpianos volares. Extirpamos todo el es-

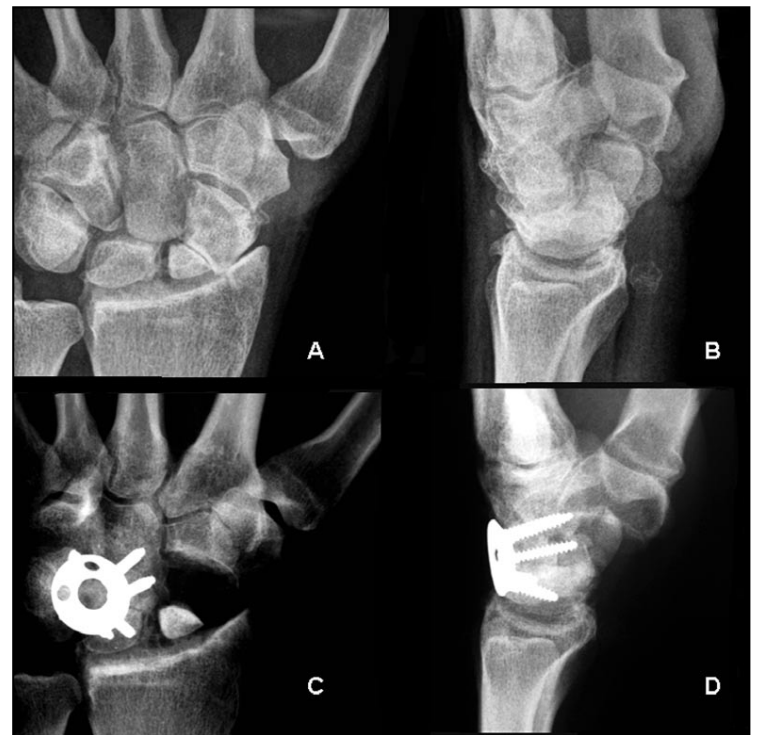

Figura 3. A y B: Muñeca SNAC, radiología preoperatorio, donde se observa como se respeta la articulación entre el radio y el polo proximal del escafoides carpiano. C y D: Radiología postoperatoria donde se observa que se ha realizado una extirpación parcial del escafoides para aumentar la superficie del cóndilo carpiano resultante tras la artrodesis.

cafoides cuando existe artrosis periescafoidea como en la muñeca SLAC (Figura 2). Realizamos una extirpación parcial del escafoides, del fragmento distal, cuando existe artrosis periescafoidea distal, pero se respeta la proximal, como sucede en la muñeca SNAC (Figura 3). Dejamos el escafoides cuando no existe artrosis periescafoidea (Figura 1 y 4). Se refrescan, con una gubia, las superficies articulares del hueso grande, semilunar y ganchoso, que se van a incluir en la artrodesis, teniendo cuidado de no dañar la parte proximal de la unión semilunopiramidal. Evitamos disociar completamente ambos huesos, para evitar lesionar el fibrocartílago que cubre la unión de ambos huesos, en donde va a pivotar todo el movimiento de la muñeca (Figura 4). Tampoco cruentamos la unión escafolunar cuando preservamos el polo proximal del escafoides en la muñeca SNAC (Figura 3). Se realiza una fijación temporal de los huesos carpianos con 2 ó 3 agujas de Kirschner, poniendo especial atención en la reducción de la dorsiflexión del hueso semilunar. Se utiliza un avellanador hasta llegar a hueso esponjoso en los huesos a artrodesar. Se obtiene el injerto esponjoso del escafoides extirpado y se introduce entre las superficies articulares, en 


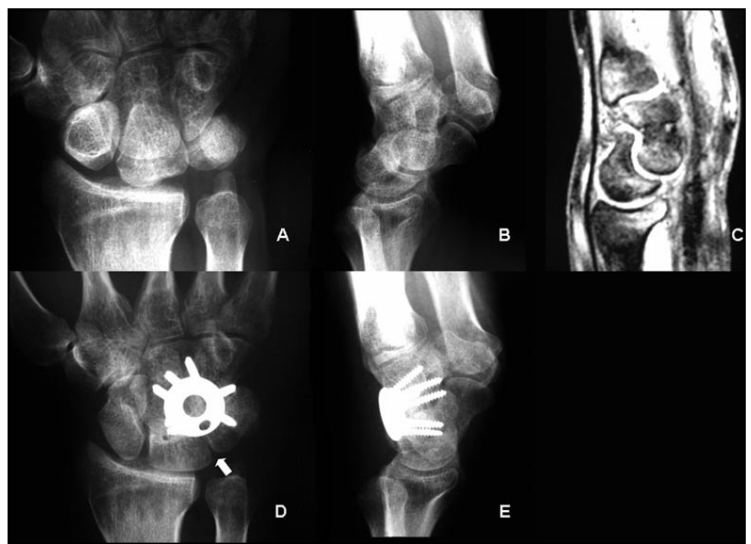

Figura 4. A y B: Imágenes radiológicas simples de una inestabilidad mediocarpiana grave. $C$ : Corte coronal de RM del mismo paciente. D y E: Control tras la artrodesis de cuatro esquinas sin extirpar el escafoides carpiano. Todas las articulaciones que rodean al escafoides están indemnes. Nótese como se respeta en la artrodesis la unión entre el semilunar y el piramidal, para no dañar la unión ligamentosa y cartilaginosa proximal, y dejar un cóndilo carpiano liso (señalado por una flecha). En la proyección lateral se observa una correcta alineación de la unión entre el semilunar y el hueso grande corrigiendo el VISI.

la unión de la fusión de las cuatro esquinas, en el fondo del defecto raspado. Si el escafoides está muy escleroso, como sucede con frecuencia en la muñeca SLAC (Figura 2A y 2B), o si se conserva parcialmente (muñeca SNAC), o totalmente, recurrimos con frecuencia a injerto esponjoso de cresta iliaca. Posteriormente se alinea la placa con precaución de que el borde proximal de la misma no protruya y origine un choque con el reborde dorsal del radio. Se fija posteriormente con tornillos, con cuidado tanto en el brocado como en la medición de la longitud de los tornillos destinados al hueso piramidal. Esto se debe a que una longitud o brocado excesivo puede ocasionar una lesión condral de la articulación piso-piramidal. Después de colocar la placa, volvemos a introducir abundante injerto esponjoso por el orificio central de la placa para no dejar ningún espacio sin rellenar (Figura 5). Se realiza control fluoroscópico para comprobar la posición de la placa y los tornillos, así como pruebas de movilidad para comprobar la estabilidad de la placa. Se cierra por planos y se inmoviliza con una férula durante tres semanas, para que cicatricen las partes blandas y comience la consolidación ósea.

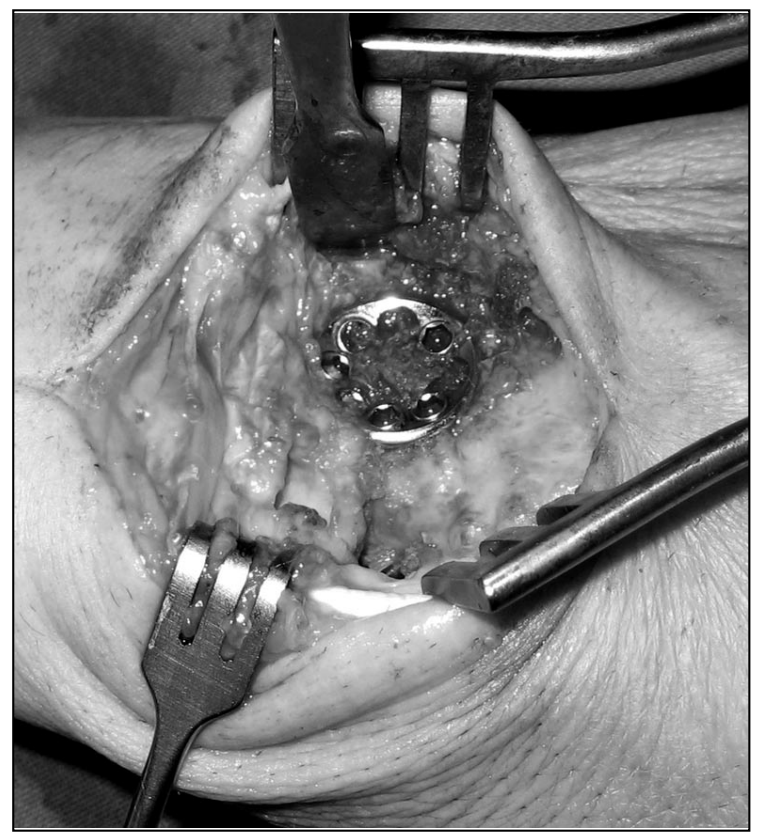

Figura 5. Campo quirúrgico donde se aprecia la placa en su posición, los intersticios óseos y el centro de la placa bien relleno de virutas de hueso esponjoso.

\section{RESULTADOS}

Al final del seguimiento (seguimiento medio 18 meses $(R=8-25)$. Todos los pacientes estaban satisfechos con su resultado en relación con alivio del dolor, movilidad de la muñeca y función. La escala visual analógica del dolor (VAS) pasó de ser de 7,3 prequirúrgica a 2,6 postquirúrgica. De los 18 pacientes trabajadores manuales, 17 se reinsertaron a su actividad profesional habitual tras la cirugía. Solo un paciente cambió de actividad laboral tras la misma.

La flexión media de la muñeca pasó de ser de $32^{\circ}\left(\mathrm{R}=20-45^{\circ}\right)$ preoperatoria a $34^{\circ}$ $(\mathrm{R}=12-45)$ postoperatorio. La extensión de la muñeca pasó de $36^{\circ}\left(\mathrm{R}=12-48^{\circ}\right)$ a $29^{\circ}(\mathrm{R}=$ $\left.12-45^{\circ}\right)$. Por lo tanto el arco de flexoextensión disminuyó de $68^{\circ}$, de promedio, en el preoperatorio a $63^{\circ}$ en el postoperatorio, pero manteniendo una situación funcional. La desviación radial preoperatoria fue de $10^{\circ}\left(\mathrm{R}=0-15^{\circ}\right)$ y la postoperatoria de $12^{\circ}\left(\mathrm{R}=0-16^{\circ}\right)$. La desviación cubital pasó de ser de $20^{\circ}\left(\mathrm{R}=5-25^{\circ}\right)$ prequirúrgica a $17^{\circ}\left(\mathrm{R}=0-20^{\circ}\right)$ postquirúrgica. El arco radiocubital se mantuvo en $29-30^{\circ}$.

Tras la cirugía, se produjo un incremento de la fuerza de puño, pasando de una media pre- 
quirúrgica de $22 \mathrm{~kg}$. ( $\mathrm{R}=12-36)$ a $25 \mathrm{~kg}$. $(\mathrm{R}=$ 10-45) en el postoperatorio.

Se consiguió la consolidación de la artrodesis en el $100 \%$ de los casos. Se apreció una leve disminución del índice de altura del carpo, pasando de ser 0,46 $(\mathrm{R}=0,35-0,51)$ prequirúrgico a $0,44(\mathrm{R}=0,35-0,50)$ tras la cirugía. $\mathrm{Al}$ final del seguimiento no se evidenciaron, con estudio radiológico, cambios degenerativos de la articulación radiosemilunar.

No hubo ninguna infección profunda ni superficial. No hubo ningún caso de síndrome de dolor regional complejo (SDRC). En uno de los pacientes de la serie presentó intenso dolor pisopiramidal, que cedía con la infiltración anestésica articular. En un TAC posquirúrgico no se observó una clara afectación articular ni protrusión del material de osteosíntesis. Fue reintervenido realizándose extirpación del hueso pisiforme resolviendo el cuadro doloroso que se atribuyó a un daño articular por el brocado del tornillo dirigido al piramidal y se obtuvo un resultado funcional final satisfactorio.

El resultado se valoraron según los siguientes criterios: Excelente si no existe dolor en las actividades diarias; el rango de movimiento es igual o superior de $45^{\circ}$ de arco de flexo-extensión y $25^{\circ}$ de arco radio-cubital; si existe incremento objetivo de la fuerza; y no han surgido complicaciones. Diecisiete pacientes cumplían estos requisitos. Tres pacientes se valoraron como resultado bueno debido a la presencia de dolor leve o la complicación menor comentada.

\section{DISCUSIÓN}

La artrodesis de las cuatro esquinas es una técnica que ha mostrado su efectividad, tanto en la estabilización de la articulación mediocarpiana, como en la eliminación del dolor en las áreas afectadas. Las únicas dos contraindicaciones absolutas son la translación cubital y la existencia de cambios degenerativos en la articulación entre el radio y el semilunar ${ }^{4}$. Las publicaciones previas con relación a esta técnica quirúrgica notifican una preservación de la fuerza y un rango de movilidad aceptable ${ }^{4-8}$. Algunos autores realizan una artroscopia preopera- toria para evaluar el estado de los compartimentos afectados ${ }^{7}$. Nosotros no la utilizamos sistemáticamente y pensamos que añade poca información a la radiología simple siendo únicamente justificable cuando se sospeche patología asociada del fibrocartílago triangular.

La clave del éxito de esta técnica quirúrgica depende de la obtención de una fusión sólida entre los cuatro huesos, con el semilunar en una posición adecuada neutra. Esto permite por lo tanto la supresión del dolor y conseguir el máximo de movilidad en el espacio radiocarpiano $^{1,9}$. El mantenimiento del máximo rango de movilidad tras esta operación se basa en una buena técnica quirúrgica con corrección de la desaxaciones y de un adecuado programa de rehabilitación de la muñeca, que debe ser de inicio precoz y de duración prolongada.

Para realizar una rehabilitación precoz es preciso obtener una estabilización inmediata. Con este objetivo empleamos una modificación técnica con un injerto corticoesponjoso atornillado $^{2}$. Animados por los resultados obtenidos empleamos la placa Spider con el mismo propósito. ${ }^{10} \mathrm{La}$ movilización precoz también contribuye a disminuir la incidencia de SDRC, que no observamos en ningún caso, pero tiene una incidencia del $1,5 \%$ en las grandes series publicadas. ${ }^{8}$ La fijación interna con el uso de un dispositivo mínimo reduce el riesgo de infección y además evita las molestias originadas al paciente con la retirada de las agujas.

No siempre se necesita la extirpación del escafoides carpiano. Su extirpación es imprescindible en los casos de colapso con artrosis periescafoidea, tal como describió Watson et al. ${ }^{1}$, para resolver el dolor generado en las articulaciones artrósicas alrededor del escafoides. Pero existen indicaciones de la artrodesis de las cuatro esquinas donde las articulaciones periescafoideas están indemnes, como suceden en la necrosis avascular de la cabeza del hueso grande, las lesiones osteocondrales traumáticas de la cabeza del hueso grande (Figuras 1 y 2) y la inestabilidad mediocarpiana (Figura 5). En la muñeca SNAC, donde la articulación entre el polo proximal del escafoides carpiano y la fosa escafoidea del radio esta preservada, se puede dejar en su posición el polo proximal del escafoides que aumentará la superficie articu- 
lar del nuevo cóndilo carpiano creado tras la artrodesis (Figura 4).

En el estudio radiológico observamos una conservación de la altura del carpo y consolidación de todos los casos. Esta puede ser difícil de valorar, especialmente cuando se coloca un dispositivo circular metálico que no permite visualizar completamente los espacios entre los 4 huesos a artrodesar en la proyección anteroposterior. En la lateral existe un solapamiento de los huesos carpianos que puede dar una imagen de falsa fusión. Por esta razón hay que tener en cuenta también la integración del dispositivo de fijación interna, la falta de osteolisis alrededor de los tornillos, la no presencia de rotura de material y por supuesto cotejarlo con la clínica. Para solventar este problema han salido también al mercado placas radiotransparentes. Algunos autores han propuesto la realización de un TAC en los casos dudosos para valorar la consolidación ${ }^{11}$.

Los resultados muestran una preservación del rango de movilidad. La pérdida de extensión se compensa con un mantenimiento de la flexión. La disminución de la desviación cubital se compensa con un incremento de la desviación radial. Cuando comparamos nuestros resultados con otras series, hay que resaltar nuestro éxito en el control del intenso dolor preoperatorio $^{4,5,7,8}$ Esto depende principalmente de conseguir la consolidación, mantener indemne la parte más proximal de la unión semiluno-piramidal, la extirpación del escafoides deteriorado cuando es necesario, la asociación de la neurectomía del nervio interóseo posterior y por supuesto de la selección de los pacientes más sintomáticos.
Estudios recientes reportan una elevada incidencia de seudoartrosis $(25 \%)$ en las artrodesis realizadas con placa circular ${ }^{12,13}$. Esto contrasta con los buenos resultados observados en nuestra serie. Esta divergencia se puede justificar porque siempre realizamos un cruentado minucioso de las superficies articulares de los huesos a artrodesar y realizamos un relleno de todos los espacios existentes con injerto de esponjosa (Figura 6). Como el escafoides con frecuencia esta escleroso proporciona un injerto de mala calidad, por lo que en estos casos, o cuando se preserva este hueso, recurrimos al injerto esponjoso de cresta ilíaca. Por otro lado siempre mantenemos una inmovilización durante 3 semanas para que cicatricen las partes blandas y comience la consolidación. Luego se sigue con una rehabilitación progresiva dirigida.

Podemos concluir, sobre la base de nuestra experiencia y a nuestros resultados, que la artrodesis de las cuatro esquinas esta indicada en los casos sintomáticos de muñeca SLAC o SNAC grados II y III, en la necrosis avascular de la cabeza del hueso grande con colapso articular mediocarpiano, en las lesiones osteocondrales traumáticas de la cabeza del hueso grande y en la inestabilidad mediocarpiana. El uso de placas circulares satisface los objetivos previamente establecidos de fijación inmediata estable, movilización temprana, alto índice de consolidaciones y preservación de la altura del carpo. La utilización de placas circulares permite una movilización y rehabilitación más precoz pero hay que hacer un cruentado minucioso de todas las superficies articulares y un relleno de injerto con astillas de tejido esponjoso. 


\section{BIBLIOGRAFÍA}

1. Watson HK, Ballet FL. The SLAC wrist: scapholunate advanced collapse pattern of degenerative arthritis. J Hand Surg A, 1984; 9: 358-65.

2. García-López A, Pérez-Ubeda MJ, Marco F, et al. A modified technique of four-bone fusion for advanced carpal collapse (SALAC/SNAC wrist). J Hand Surg B, 2001; 26: 352-4.

3. Mc Murtry R, Youm Y, Flatt A, et al. Kinematics of the wrist. Part. II: Clinical applications. J Bone Joint Surg A, 1978; 60: 955-61.

4. Ashmead D, Watson HK, Damon C, et al. Scapholunate advanced collapse wrist salvage. J Hand Surg A, 1994; 19: 741-50.

5. Krakauer JD, Bishop AT, Cooney WP. Surgical treatment of scapholunate advanced co- llapse. J Hand Surg A,1994; 19: 751-9.

6. Sauerbier M, Tränkle M, Bickert $B$, et al. Outcome assessment of midcarpal arthrodesis with complete scaphoid excision in the treatment of advanced carpal collapse (SNAC/SLAC wrist). J Hand Surg B, 2000; 25 (suppl 1): 64.

7. Voche Ph, Merle M. Four-bone fusion of the wrist. A review of 12 cases. Rev Chir Orthop, 1993; 79: 456-63.

8. Watson HK, Weinzweig J, Guidera PM, et al. One thousand intercarpal arthrodesis. J Hand Surg B, 1999; 24: 307-15.

9. Baratz ME, Towsen A. Midcarpal arthrodesis. Four-bone technique. Tech Hand Upper Extrem Surg, 1997; 1: 237-44.

10. De Francisco-Marugan B, A. García-López, Y Lópiz-Mora- les, et al. Artrodesis mediocarpiana con placa Spider. Rev Ortop Traumatol, 2007; 51: 69-71.

11. Ekelund L, Hagberg L, Horberg $\mathrm{L}$, et al. Imaging of four-corner fusion (SLAC arthrodesis) of the wrist with 64-slice computed tomography. Acta Radiol, 2007; 48: 76-9.

12. Chung KC, Watt AJ, Kotsis SV. A prospective outcomes study of four-corner wrist arthrodesis using a circular limited wrist fusion plate for stage II scapholunate advanced collapse wrist deformity. Plast Reconstr Surg, 2006; 118: 433-42.

13. Shindle MK, Burton KJ, Weiland $\mathrm{AJ}$, et al. Complications of circular plate fixation for four-corner arthrodesis. J Hand Surg B, 2007; 32: 50-3. 